\title{
Sur l'équation de Lamé
}

(extrait d'une lettre à Mr. Brioschi).

(Par Mr. C. Hermite, à Paris.)

Vous ne serez donc pas surpris que je sois parvenu de mon côté à l'équation différentielle du $3^{\text {me }}$ ordre:

$$
z^{\prime \prime \prime}+3 p z^{\prime \prime}+\left(p^{\prime}+2 p^{2}+4 q\right) z^{\prime}+2\left(q^{\prime}+2 p q\right) z=0
$$

dont les solutions sont les produits de deux solutions de l'équation du second ordre:

$$
y^{\prime \prime}+p y^{\prime}+q y=0
$$

mais je l'obtiens sous une forme un peu différente, en prenant pour point de départ, l'équation:

$$
2 A y^{\prime \prime}+A^{\prime} y^{\prime}=B y
$$

Un calcul facile me donne:

$$
2 A z^{\prime \prime}+3 A^{\prime} z^{\prime \prime}+A^{\prime \prime} z^{\prime}=4 B z^{\prime}+2 B^{\prime} z
$$

et voici les conséquences que j'en tire. Faisant dans l'équation de Lamé, $s n^{2} x=t$, on obtiendra pour transformée l'équation (1), on l'on prendra:

$$
\begin{aligned}
A & =t(1-t)\left(1-k^{2} t\right) \\
2 B & =n(n+1) k^{2} t+h .
\end{aligned}
$$

Les fonctions $A$ et $B$ étant ainsi de simples polynômes, du troisième et du premier degré en $t$, la différentiation d'ordre $p$ de l'équation (2), donne:

$$
\begin{gathered}
2 A z^{p+3}+(2 p+3) A^{\prime} z^{p+2}+\left[\frac{5 p(p+1)+2}{2} A^{n}-2 B\right] z^{p+1} \\
+\left[2 p(p-1)(p-2)+9 p(p-1)+6 p-(2 p+1)\left(n^{2}+n\right)\right] k^{2} z^{p}=0 ;
\end{gathered}
$$


or on peut mettre le coefficient de $z^{p}$, sous la forme:

$$
(2 p+1)(p-n)(p+n+1)
$$

il s'annulle donc en faisant $p=n$, et en adoptant cette valeur, l'équation est satisfaite si l'on pose $z^{p}=$ const. L'équation (2) par conséquent admet pour solution un polynôme entier en $t$ de degré $n, z=F(t)$, et les conclusions aux qu'elles vous êtes parvenu pour $n=1$, s'étendent d'elles mêmes au cas ou $n$ est quelconque. En effet, deux solutions $y_{1}$ et $y_{2}$ de l'équation (1) sont liées par la relation:

$$
y_{2} \frac{d y_{1}}{d t}-y_{1} \frac{d y_{2}}{d t}==\frac{C}{\sqrt{A}}
$$

ou $C$ est une constante, et en y joignant la condition:

$$
\frac{d\left(y_{1} y_{2}\right)}{d t}=y_{2} \frac{d y_{1}}{d t}+y_{1} \frac{d y_{2}}{d t}=F^{\prime}(t)
$$

on en déduira:

$$
y_{2} \frac{d y_{1}}{d t}=\frac{1}{2}\left[F^{\prime}(t)+\frac{C}{\sqrt{ } A}\right], \quad y_{1} \frac{d y_{2}}{d t}=\frac{1}{2}\left[F^{\prime}(t)-\frac{C}{\sqrt{ } A}\right]
$$

et par suite:

$$
\frac{1}{y_{1}} \frac{d y_{1}}{d t}=\frac{1}{2}\left[\frac{F^{\prime}(t)}{F^{\prime}(t)}+\frac{C}{\sqrt{A F(t)}}\right], \quad \frac{1}{y_{2}} \frac{d y_{2}}{d t}=\frac{1}{2}\left[\frac{F^{\prime}(t)}{F(t)}-\frac{C}{\sqrt{A F(t)}}\right]
$$

d'où enfin :

$$
y=G e^{\frac{1}{2} \int\left[\frac{F^{\prime}(t)}{F^{\prime}(t)}+\frac{C}{\sqrt{A F(t)}}\right] d t}+G^{\prime} e^{\frac{1}{2} \int\left[\frac{F^{\prime}(t)}{\bar{F}(t)}-\frac{C}{\sqrt{A F(t)}}\right] d t}
$$

en désignant par $G$ et $G^{\prime}$ deux constantes arbitraires.

Voici maintenant à l'égard de la costante $C$, une remarque essentielle. On tire aisément de l'équation (2), la suivante:

$$
A\left(2 z z^{\prime \prime}-z^{\prime 2}\right)+A^{\prime} z z^{\prime}=2 B z^{2}-N
$$

et en résultat se vérifie sur le champ en différentiant et divisant les deux membres par $z$. Mais à la solution spéciale de cette équation qui est donnée en prenant pour $z$ le polynôme $F(t)$, correspond une valeur entièrement déterminée de $N$. Qu' on attribue en effet à la variable $t$ pour valeur particulière une racine de l'équation $y_{1}=0$, nous aurons en même temps, $z=0, z^{\prime}=y_{1}^{\prime} y_{2}$, 
donc: $N=A\left(y_{1}^{\prime} y_{2}\right)^{2}$. Or en attribuant cette même valeur à $t$, dans l'équation

$$
y_{2} \frac{d y_{1}}{d t}-y_{1} \frac{d y_{2}}{d t}=\frac{C}{\sqrt{A}}
$$

vous voyez qu'on en conclut: $C=\sqrt{ } A y_{1}^{\prime} y_{2} ;$ nous parvenons par suite à cette expression $C=\sqrt{N}$, et tout se trouve par conséquent déterminé dans la formule (3) qui donne ainsi la solution complète de l'équation de Lamé.

Vous reconnaitrez maintenant sans peine, qu'en posant $N=0$ on a les raleurs particulières de $h$ aux quelles correspondent les solutions qui, à l'égard de la variable $x$, sont des fonctions doublement périodiques, mais en laissant de côté, ce point, je vous indiquerai une dernière remarque. L'équation (4) montre qu'en supposant $N$ différent de zéro, il est impossible d'avoir à la fois: $F(t)=0$, et $F^{\prime}(t)=0$, de sorte que la première équation n'a que des racines simples. Soit: $t=\tau$ l'une quelconque de ces racines, et faisons:

$$
\frac{1}{F(t)}=\mathbf{\Sigma} \frac{1}{F^{\prime \prime}(\tau)(t-\tau)}
$$

Si nous désignons par $T$ la valeur de $A$ pour $t=\tau$, de sorte que l'équation (4), donne:

$$
T F^{\prime 2}(\tau)=N
$$

on en conclura:

$$
\frac{\sqrt{N}}{F(t)}=\mathbf{\Sigma} \frac{\sqrt{N}}{F^{\prime}(\tau)(t-\tau)}=\mathbf{\Sigma} \frac{\sqrt{T}}{t-\tau}
$$

et par conséquent:

$$
\frac{F^{\prime}(t)}{F(t)}+\frac{\sqrt{N}}{\sqrt{A F(t)}}=\Sigma\left[\frac{1}{t-\tau}+\frac{\sqrt{T}}{\sqrt{A(t-\tau)}}\right]=\Sigma \frac{\sqrt{A}+\sqrt{T}}{\sqrt{A(t-\tau)}}
$$

Cette formule conduit de la manière la plus facile à l'expression de l'intégrale qui figure en exponentielle dans l'équation (3). Faisant en effet: $t=s n^{2} x$, $\tau=s n^{2} \omega$, on $\mathrm{a}$ :

$$
\frac{1}{2} \int \frac{\sqrt{A}+\sqrt{T}}{t-\tau} d t=\int \frac{\operatorname{sn} x \operatorname{cn} x d n x+\operatorname{sn} \omega \operatorname{cn} \omega d n \omega}{\operatorname{sn}^{2} x-\sin ^{2} \omega} d x=\int\left[\frac{H^{\prime}(x-\omega)}{H(x-\omega)}-\frac{\Theta^{\prime}(x)}{\Theta(x)}+\frac{\Theta^{\prime}(\omega)}{\Theta(\omega)}\right] d x \text {. }
$$

(voyez C. R., pag. 1086). Soit pour plus de clarté: $\omega_{1}, \omega_{2}, \ldots \omega_{n}$ les $n$ détermination de $\omega$ qui correspondent aux diverses racines $\tau$, et qui ont été choisies

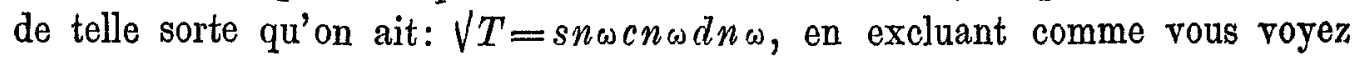


la supposition: $\vee T=-s n \omega c n \omega d n \omega$, nous parvenons à ce résultat:

$$
e^{\frac{1}{2} \int\left[\frac{F^{\prime}(t)}{F^{\prime}(t)}+\frac{\sqrt{N}}{\sqrt{A} \vec{F}^{\prime}(t)}\right] d t}=\frac{H\left(x-\omega_{1}\right) H\left(x-\omega_{2}\right) \cdots H\left(x-\omega_{n}\right)}{\theta^{n}(x)} e^{x \sum \frac{\theta^{\prime}(\omega)}{\Theta(\omega)}}
$$

et il est clair qu' on aurait semblablement:

$$
e^{\frac{1}{2} \int\left[\frac{F^{\prime}(t)}{F^{\prime}(t)}-\frac{\sqrt{N}}{\sqrt{A F(t)}}\right] d t}=\frac{H\left(x+\omega_{1}\right) H\left(x+\omega_{2}\right) \cdots H\left(x+\omega_{n}\right)}{\Theta^{n}(x)} e^{-x \Sigma \frac{\Theta^{\prime}(\omega)-}{\Theta(\omega)}}
$$

Cette méthode pour intégrer l'équation de Lamé se trouve dans les feuilles lithographiées de mon cours de 1872 à l'Ecole Polytecnique . . . . . .

17 décembre 1877. 